\title{
Peroxisome proliferator-activated receptor $\gamma$ agonist rosiglitazone inhibits migration and invasion of prostate cancer cells through inhibition of the CXCR4/CXCL12 axis
}

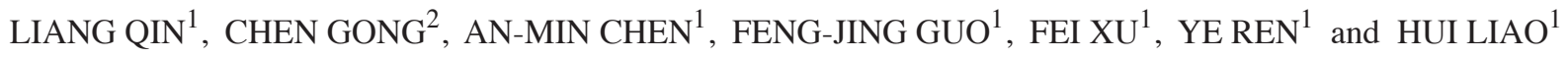 \\ Departments of ${ }^{1}$ Orthopedics and ${ }^{2}$ Oncology, Tongji Hospital, Tongji Medical College, \\ Huazhong University of Science and Technology, Wuhan, Hubei 430030, P.R. China
}

Received July 30, 2013; Accepted March 7, 2014

DOI: $10.3892 / \mathrm{mmr} .2014 .2232$

\begin{abstract}
It has been indicated that the C-X-C chemokine receptor type 4/C-X-C chemokine ligand 12 (CXCR4/CXCL12) axis is involved in promoting invasion and metastasis in tumors. Therefore, novel drugs capable of downregulating the CXCR4/CXCL12 axis may demonstrate potential for the treatment of metastatic prostate cancer (PCa). Rosiglitazone (RSG), a thiazolidinedione ligand of the peroxisome proliferator-activated receptor (PPAR) $\gamma$, has been found to inhibit proliferation, induce apoptosis, suppress angiogenesis and inhibit metastasis. However, the precise mechanisms by which RSG regulates CXCR4 gene expression and the consequent effects on prostate cell migration and invasion are not fully understood. In this study, it was observed that RSG is capable of downregulating the expression of CXCR4 in PCa cells in a dose-, time- and PPAR $\gamma$-dependent manner. Furthermore, it was observed that the downregulation of CXCR4 expression occurred at a transcriptional level, as indicated by a reduction in CXCR4 mRNA expression. Suppression of CXCR 4 expression by RSG further correlated with the inhibition of CXCL12-induced migration and invasion in PCa cells. Analysis of the predominant intracellular signaling pathways that act downstream of the activated CXCR4/CXCL12 axis, namely the phosphatidyl inositol 3-kinase (PI3K)-protein kinase B (Akt) cascades, revealed that RSG rapidly interferes with the phosphorylation/activation of Akt, which mediates CXCL12-stimulated migration and invasion. Overall, the findings of this study suggest that RSG represents a novel inhibitor of CXCR4
\end{abstract}

Correspondence to: Dr Hui Liao, Department of Orthopedics, Tongji Hospital, Tongji Medical College, Huazhong University of Science and Technology, 1095 Jie Fang Avenue, Wuhan, Hubei 430030, P.R. China

E-mail: Liaohui@tjh.tjmu.edu.cn

Key words: peroxisome proliferator-activated receptor $\gamma, \mathrm{CXCR} 4$, prostate cancer, invasion expression and, thus, has significant potential as a powerful therapeutic agent for the treatment of metastatic PCa.

\section{Introduction}

Prostate cancer (PCa) is the second leading cause of increased cancer incidence and cancer-associated mortality among males in the United States (1). In 2010, the incidence of new prostate cancer cases was estimated to be 217,730 , resulting in 32,050 mortalities (2). The majority of patients with advanced PCa develop bone metastases and suffer from long-term skeletal morbidity, involving pain, pathological fractures and spinal cord compression, which has a significant impact on the quality of life of the patient. Therefore, studying the molecular mechanisms underlying $\mathrm{PCa}$ bone metastasis is considered essential for the prevention and treatment of PCa.

Peroxisome proliferator-activated receptor (PPAR) $\gamma$ regulates the expression of genes involved in the control of lipid metabolism and insulin sensitivity via ligand-activated transcriptional activity (3). PPAR $\gamma$ ligands include naturally occurring fatty acids, 15-deoxy-delta12,14-prostaglandin J2 (PGJ2) and thiazolidinediones (TZDs), such as troglitazone and rosiglitazone (RSG) (4). The activation of PPAR $\gamma$ by TZDs and other ligands has been shown to inhibit proliferation and invasion, as well as induce apoptosis and cell cycle arrest, in prostate and other cancer cells through PPAR $\gamma$-dependent and -independent pathways (5-9). Therefore, PPAR $\gamma$ is recognized as a relevant target for cancer therapy.

The present study aimed to investigate the effect of PPAR $\gamma$ activation by ligands on tumor cell migration and invasion. C-X-C chemokine receptor type 4 (CXCR4) has been reported to mediate proliferation, invasion and metastasis of tumor cells; therefore, it was hypothesized that RSG may modulate the expression of CXCR4 and inhibit the migration and invasion of prostate cancer cells.

\section{Materials and methods}

Cell culture. PC-3 human prostate carcinoma cells were initially stored frozen in our laboratory (Department of Orthopedics, Tonji Medical College, Wuhan, China) prior to 
cultivation in RPMI-1640 medium (Hyclone Laboratories, Inc., Logan, UT, USA) supplemented with $10 \%$ fetal bovine serum (Gibco-BRL, Grand Island, NY, USA), $1 \times 10^{5} \mathrm{U} / 1$ penicillin and streptomycin (Hyclone Laboratories, Inc.) in an incubator at $37^{\circ} \mathrm{C}$ with $5 \% \mathrm{CO}_{2}$.

Cell migration assay. Cell motility was assessed using an in vitro wound healing assay. PC-3 cells were plated in a six-well plate and grown until confluent. Monolayers of confluent PC-3 cells were then scarred, and the repair was monitored using an inverted microscope (Nikon TE200-S; Nikon Corporation, Tokyo, Japan), following $12 \mathrm{~h}$ pretreatment with $10 \mu \mathrm{M}$ RSG (Sigma Aldrich, St. Louis, MO, USA) then $24 \mathrm{~h}$ exposure to 100 ng/ml CXCL12 (R\&D Systems Inc., Minneapolis, MN, USA). Wound width was measured at 0 and $24 \mathrm{~h}$ following the start of incubation with or without RSG and in the absence or presence of CXCL12. Experiments were performed in triplicate and data are presented as the mean \pm standard deviation.

Cell invasion assay. The invasive potential of PC-3 cells was quantified using a Matrige ${ }^{\mathrm{TM}}$-coated Transwell system, as described previously (10). The chamber (Corning Inc., Corning, NY, USA) contained a polycarbonate membrane filter with a pore size of $8 \mu \mathrm{m}$, which was coated with Matrigel and inserted into a 24-well culture plate. PC-3 cells were then seeded in the top chamber of the Matrigel. Following pre-incubation with or without RSG $(10 \mu \mathrm{M})$ for $12 \mathrm{~h}$, Transwell chambers were then placed into the wells of a 24 well plate, in which either basal medium or basal medium supplemented with $100 \mathrm{ng} / \mathrm{ml}$ CXCL12 was added for $24 \mathrm{~h}$. After $48 \mathrm{~h}$ incubation, PC-3 cells on the upper surface of the filters were removed using cotton swabs. Cells that had invaded the lower surface of the membrane were fixed using methanol and stained with crystal violet. Each experiment was performed in triplicate and cells were counted in five fields in each well using light microscopy. The invasive ability of PC- 3 cells was assessed relative to the invasive ability of the untreated control cells.

Western blot analysis. Cells were grown on six-well culture plates, washed with $1 \mathrm{X}$ phosphate-buffered saline (PBS) and harvested in lysis buffer containing $20 \mathrm{mM}$ Tris- $\mathrm{HCl}$ (pH 7.5), $150 \mathrm{mM} \mathrm{NaCl}, 1 \mathrm{mM} \mathrm{Na}{ }_{2}$ EDTA, $1 \mathrm{mM}$ ethylene glycol tetraacetic acid (EGTA), $1 \%$ Triton, $2.5 \mathrm{mM}$ sodium pyrophosphate, $1 \mathrm{mM} \beta$-glycerophosphate, $1 \mathrm{mM} \mathrm{Na}_{3} \mathrm{VO}_{4}$, $1 \mu \mathrm{g} / \mathrm{ml}$ leupeptin and $1 \mathrm{mM}$ phenylmethanesulfonyl fluoride (Cell Signaling Technology Inc., Beverly, MA, USA). The cell extractions were collected and centrifuged at $10,000 \mathrm{x} \mathrm{g}$ for $10 \mathrm{~min}$ at $4^{\circ} \mathrm{C}$ before the supernatants were collected as cell lysates. Equal concentrations of total cell lysate were resolved using 10\% SDS-PAGE (Boster, Wuhan, China) and transferred to a polyvinylidene fluoride (PVDF) transfer membrane (Merck Millipore, Billerica, MA, USA). Non-specific binding sites were blocked using $5 \%$ non-fat dry milk in $1 \mathrm{X}$ Tris-buffered saline containing $0.1 \%$ Tween 20 (TBST; Boster), followed by incubation with primary antibodies against the proteins of interest in $3 \%$ bovine serum albumin (BSA; Boster)-TBST [phosphorylated Akt (p-Akt), CXCR4] or 5\% non-fat dry milk-TBST (Akt, $\beta$-actin). Subsequently, the membranes were incubated with an appropriate secondary antibody (horseradish peroxidase-conjugated goat anti-mouse or anti-rabbit immunoglobulin G; Wuhan Boster Biological Technology Ltd, Wuhan, China). Immunoblots were visualized using SuperSignal ${ }^{\mathrm{TM}}$ West Pico Chemiluminescent substrate (Thermo Fisher Scientific, Waltham, MA, USA). The antibodies raised against human Akt, p-Akt (Ser473) and $\beta$-actin (rabbit monoclonal IgG) were purchased from Cell Signaling Technology. The antibody raised against human CXCR4 (rabbit polyclonal IgG) were purchased from Santa Cruz Biotechnology, Inc. (Santa Cruz, CA, USA).

Quantitative polymerase chain reaction ( $q P C R)$ analysis. PC-3 cells were plated in six-well plates for all experiments and allowed to grow for $48 \mathrm{~h}$ prior to any treatment. GW9662 (Santa Cruz Biotechnology, Inc.) was added $2 \mathrm{~h}$ prior to any other treatment. Total RNA was extracted using TRIzol $^{\circledR}$ reagent according to the manufacturer's instructions (Invitrogen Life Technologies, Carlsbad, CA, USA). A total of $1 \mu \mathrm{g}$ RNA was reverse-transcribed according to the kit's instructions (Invitrogen Life Technologies). qPCR was performed using TransStart Green qPCR SuperMix (TransGen Biotech Co., Beijing, China) using an iQ5 Sequence Detection System (Bio-Rad Laboratories Inc., Berkley, CA, USA). Results were normalized to those obtained for GAPDH. Each sample was analyzed in triplicate and the experiment was repeated twice. The theshold cycle (CT) value (the cycle number at which the fluorescence crosses the threshold) was measured and $2^{-\Delta \mathrm{CT}}$ $\left(\Delta \mathrm{CT}=\mathrm{CT}-\mathrm{CT}_{\mathrm{GAPDH}}\right)$ was defined as the quantity of the amplified fragment. The primer sequences are listed in Table I.

Statistical analysis. Data are presented as the mean \pm standard deviation. Statistical analyses were performed using SPSS 11.0 statistics software (SPSS, Inc., Chicago, IL, USA). Statistical significance was analyzed using one-way analysis of variance. Where significance was observed, a Dunnett's post-hoc test was used to determine the statistical significance of the differences between the treated- and untreated-groups, with a value of $\mathrm{P}<0.05$ considered to indicate a statistically significant difference.

\section{Results}

RSG suppresses CXCR4 mRNA and protein levels in prostate cancer cells. A previous study has shown that CXCR4 expression is significantly higher in human $\mathrm{PCa}$ tissue than in hyperplastic prostate tissues (11). This finding suggests that CXCL12 may exhibit an autocrine regulatory role via its receptor, CXCR4, in the regulation of PCa cell migration, invasion and metastasis (12). Therefore, the initial investigations in the present study focused on the effect of RSG on the expression of CXCR4 in PC-3 cells. It has been reported that TZDs activate PPAR $\alpha$ and PPAR $\delta$ receptors at concentrations $>10 \mu \mathrm{M}$ (13); therefore, concentrations of $\leq 10 \mu \mathrm{M}$ were utilized in the present investigations. It was observed that when PC-3 cells were incubated with various RSG concentrations for $24 \mathrm{~h}$, or for various time periods with $10 \mu \mathrm{M} \mathrm{RSG}$, the expression of CXCR4 was suppressed in a dose- and time- dependent manner, respectively (Fig. 1A and B). This downregulation was not due to a decrease in cell viability, as $\sim 90 \%$ of PC- 3 cells were viable under these conditions (data not shown). 
Table I. Primer sequences for qPCR analysis.

\begin{tabular}{lll} 
Gene & \multicolumn{1}{c}{ Forward (5'-3') } & \multicolumn{1}{c}{ Reverse (5'-3') } \\
\hline GAPDH & CCATGAGAAGTATGACAACAGCC & GGGTGCTAAGCAGTTGGTG \\
CXCR4 & TGCCCACCATCTACTCCATCA & AGGATGACCAATCCATTGCCC \\
VEGF & TTACGGTCTGTGTCCAGTGTA & TTCTCTGTTATGTTGCCAGCC \\
MMP2 & GATACCCCTTTGACGGTAAGGA & CCTTCTCCCAAGGTCCATAGC \\
MMP9 & TGTACCGCTATGGTTACACTCG & GGCAGGGACAGTTGCTTCT
\end{tabular}

CXCR4, C-X-C chemokine receptor type 4; VEGF, vascular endothelial growth factor; MMP, matrix metalloproteinase.

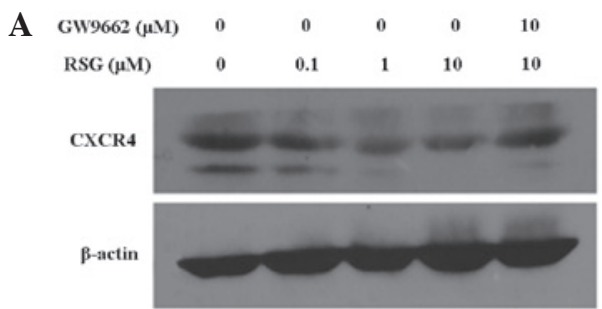

C

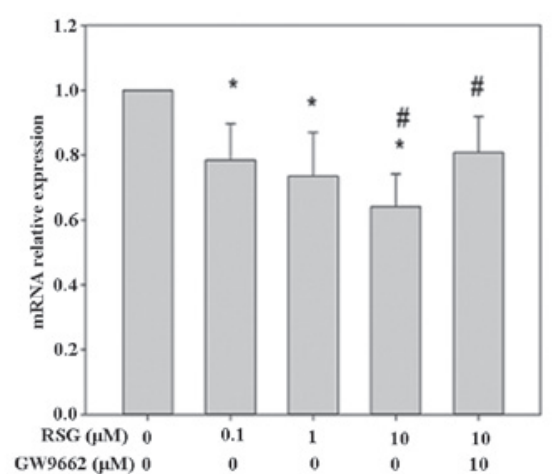

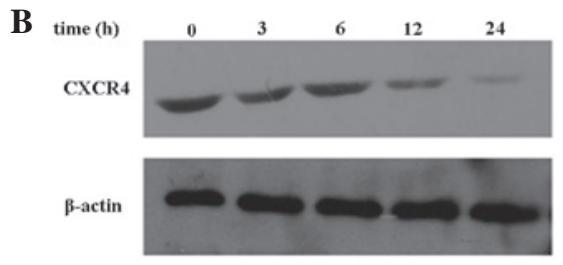

D

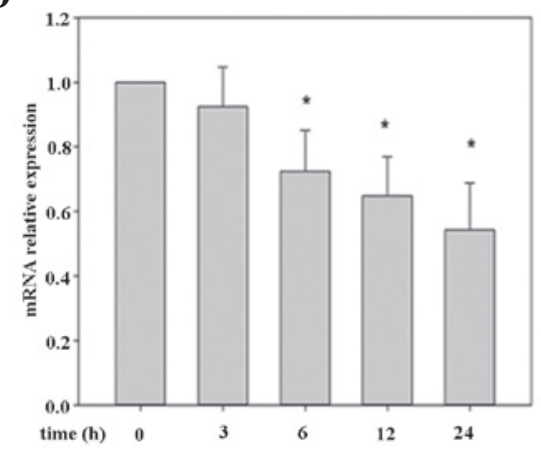

Figure 1. RSG suppresses CXCR4 mRNA and protein levels in prostate cancer cells. PC-3 cells were treated with (A) the indicated concentrations of RSG for $12 \mathrm{~h}$ or (B) $10 \mu \mathrm{M}$ RSG for the indicated times. The expression of CXCR4 was determined using SDS-PAGE and western blotting. $\beta$-actin was used as a loading control. Following incubation with either (C) various concentrations of RSG for $12 \mathrm{~h}$ or (D) $10 \mu \mathrm{M}$ RSG for various durations, total RNA was extracted from PC-3 cells and CXCR4 expression was measured using qPCR. CXCR4 expression was quantified using GAPDH as a normalization control. Data are presented as the mean \pm standard deviation of three independent experiments. ${ }^{*} \mathrm{P}<0.05$, compared with the untreated control cells; ${ }^{*} \mathrm{P}<0.05$, GW9662 pretreatment group compared with the $10 \mu \mathrm{M}$ RSG treatment group. RSG, rosiglitazone; CXCR4, C-X-C chemokine receptor type 4; qPCR, quantitative polymerase chain reaction.

It was hypothesized that the suppression of CXCR4 expression may occur at the transcriptional level. Therefore, following PC-3 cell treatment with RSG for various time-periods, the mRNA was extracted for qPCR analysis. As shown in Fig. 1D, RSG was found to downregulate CXCR4 mRNA expression in a time-dependent manner, with a significant reduction observed from $6 \mathrm{~h}$ following exposure. CXCR4 mRNA expression was inhibited at all RSG doses tested, with 22, 27 and 36\% inhibition observed following $12 \mathrm{~h}$ exposure to $0.1,1$ and $10 \mu \mathrm{M}$ RSG, respectively (Fig 1C).

Although RSG was found to affect CXCR4 expression in PC-3 cells, the mechanism by which this was achieved was unclear. To determine whether the reduction in expression of CXCR4 by RSG was dependent upon the activation of PPAR $\gamma$, PC-3 cells were treated with the PPAR $\gamma$ antagonist, GW9662. The downregulation of CXCR4 mRNA and protein expression induced by RSG in PC-3 cells was attenuated upon addition of GW9662 (Fig. 1). These results indicate that RSG is capable of downregulating CXCR4 expression in a manner dependent upon PPAR $\gamma$ activation. In combination, these data suggest that RSG may inhibit CXCR4 expression in PC-3 cells, in a PPAR $\gamma$-dependent manner.

RSG inhibits CXCL12-induced migration in PC-3 cells. This study further investigated a potential correlation between RSG-induced downregulation of CXCR4 and PCa cell migration. An in vitro wound healing assay revealed that PC-3 cells migrated more rapidly upon treatment with CXCL12 and that this effect was abolished upon treatment with RSG (Fig. 2).

RSG inhibits CXCL12-induced invasion in PC-3 cells. To elucidate the inhibitory effect of RSG upon CXCL12-induced invasion in PC-3 cells, chamber invasion assays were performed. RSG was observed to suppress the invasion of PC-3 cells across the Matrigel-coated filter in a dose-dependent 
A
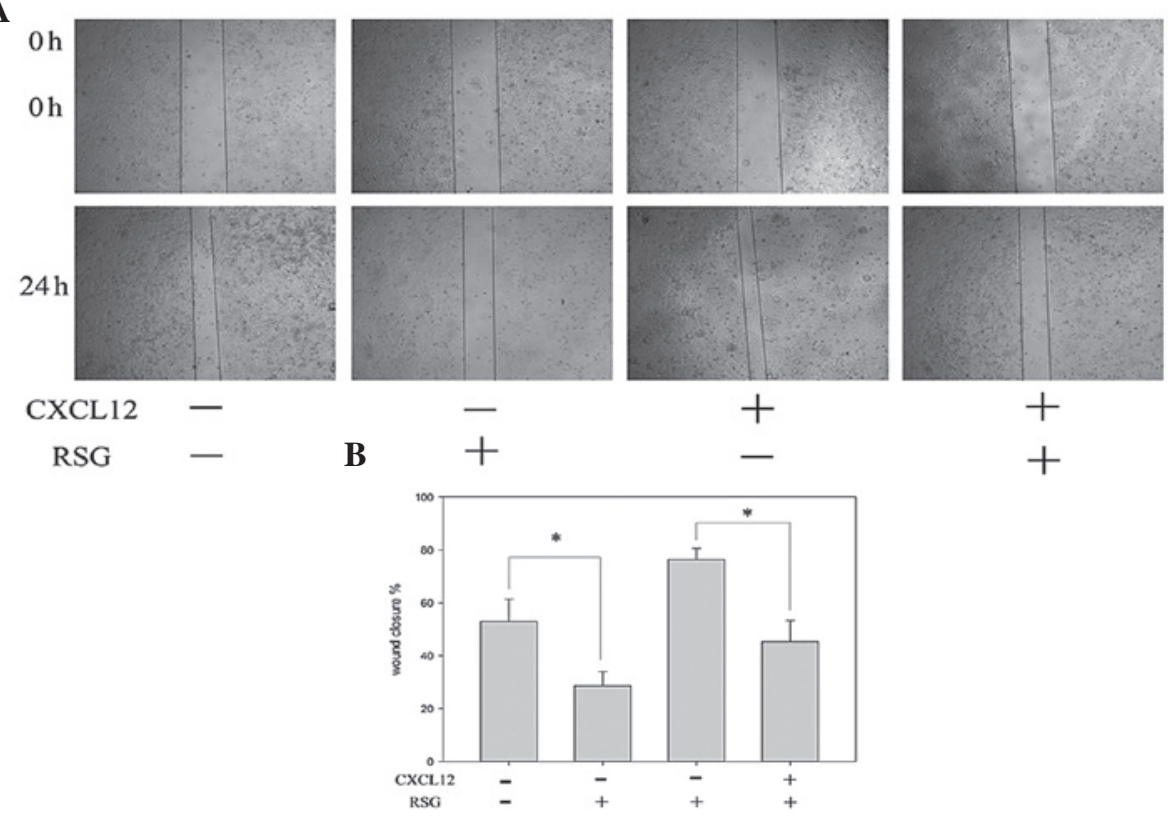

Figure 2. RSG suppresses migration of prostate cancer cells. (A) Cells that migrated to the wounded region were photographed (magnification, x40). (B) The wounded area of the cell cultures was quantified in four fields for each treatment group, and data were calculated from three independent experiments. Data are presented as the mean \pm standard deviation of three independent experiments. " $\mathrm{P}<0.05$, compared with the untreated control cells. RSG, rosiglitazone; CXCL12, C-X-C chemokine ligand 12.

A
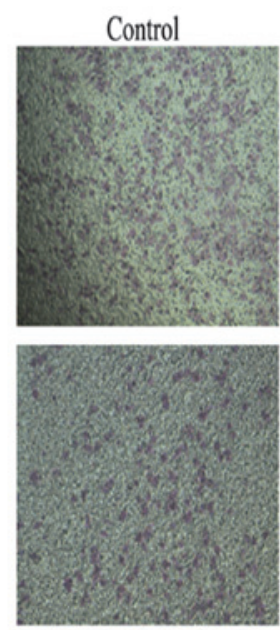

RSG
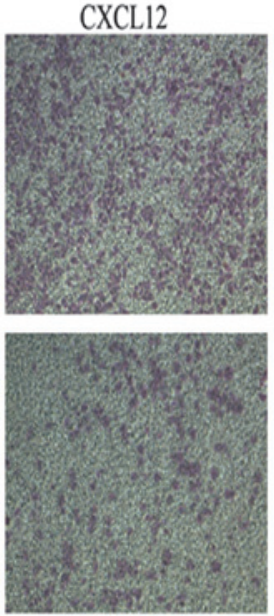

RSG+CXCL12
B

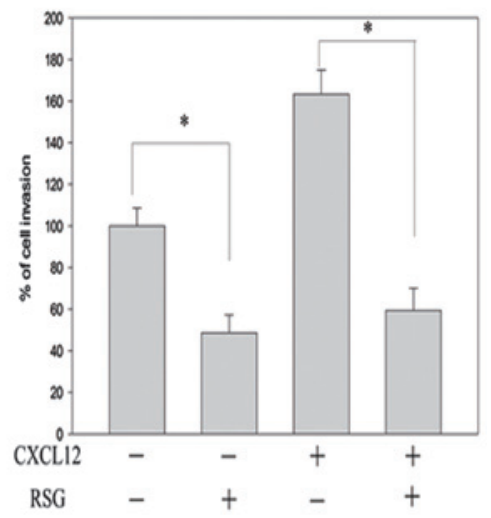

Figure 3. RSG suppresses invasion of prostate cancer cells. (A) Invaded cells were photographed (magnification, x100). (B) Invaded PC-3 cells were counted in five random fields in each treatment group, and data were calculated from three independent experiments. Data are presented as the mean \pm standard deviation of three independent experiments. " $\mathrm{P}<0.05$, compared with the untreated control group. RSG, rosiglitazone; CXCL12, C-X-C chemokine ligand 12.

manner, with $10 \mu \mathrm{M}$ RSG found to inhibit $52 \%$ of cell invasion. RSG was also observed to suppress the CXCL12-induced invasion of the PC-3 cells (Fig. 3). These results indicate that RSG markedly inhibits the CXCL12-induced invasion of PC-3 cells.

RSG inhibits CXCL12-induced Akt activation in prostate cancer cells. It has previously been indicated that the signaling proteins, PI3K and Akt, may be associated with the expression of matrix-metalloproteinases (MMPs) and metastasis induction (14). To further elucidate the signal transduction pathways responsible for CXCR4 expression and PC-3 cell migration and invasion, the activation of Akt, a signaling component of pathways coupled to G-protein-coupled chemoattractant receptors, was examined. Upon rapid stimulation $(30 \mathrm{~min})$ of PC-3 cells with $100 \mathrm{ng} / \mathrm{ml} \mathrm{CXCL12,} \mathrm{an} \mathrm{increase} \mathrm{in} \mathrm{Akt} \mathrm{phos-}$ phorylation at Ser473 was observed, resulting in the activation of the enzyme. Furthermore, it was observed that concomitant addition of $10 \mu \mathrm{M}$ RSG for 30 min downregulated the CXCL12-induced phosphorylation of Akt in a dose-dependent manner in PC-3 cells (Fig. 4A). This finding suggests that the inhibition of migration and invasion associated with RSG treatment in PC-3 cells may partly occur through the suppression of PI3K pathways. 
A

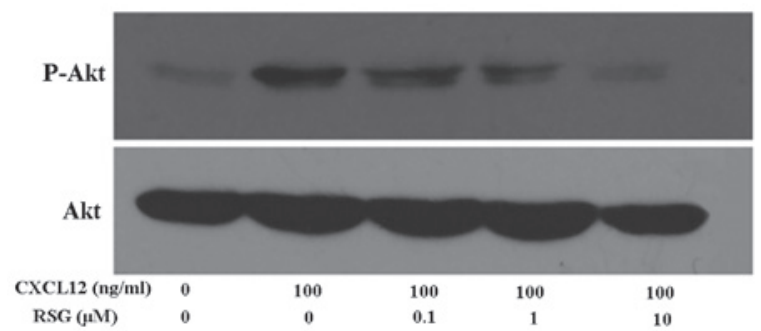

B

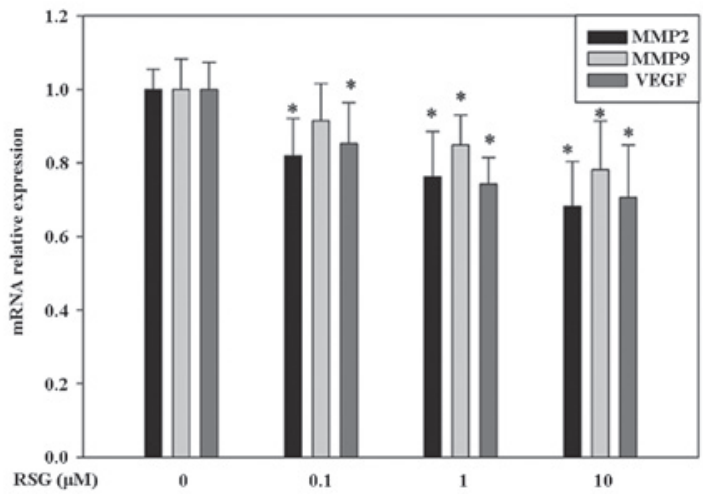

Figure 4. RSG inhibits CXCL12-induced Akt activation in prostate cance cells. (A) Western blot analysis of PC-3 cell lysates following $15 \mathrm{~min}$ stimulation with $100 \mathrm{ng} / \mathrm{ml} \mathrm{CXCL12} \mathrm{and} 10 \mu \mathrm{M}$ RSG. Phosphorylation of Akt at Ser473 is increased following CXCL12 stimulation, and is reverted by RSG treatment. Akt protein normalization is shown in the lower panel (B) The expression of each target gene was quantified using GAPDH as a normalization control. The data represent the results from three independent experiments. Data are presented as the mean \pm standard deviation of three independent experiments. ${ }^{*} \mathrm{P}<0.05$, compared with the untreated contro group. RSG, rosiglitazone; CXCL12, C-X-C chemokine ligand 12; Akt, protein kinase B; P-Akt, phosphorylated Akt; MMP, matrix metalloproteinase; VEGF, vascular endothelial growth factor.

In addition to the PI3K/Akt pathway, other signaling pathways downstream of the activated CXCR4/CXCL12 axis were investigated. PC-3 cells were treated with various concentrations of RSG for $12 \mathrm{~h}$ and the expression of MMP-2, MMP-9 and vascular endothelial growth factor (VEGF) mRNA were then analyzed using qPCR, respectively. As demonstrated in Fig. 4, RSG significantly downregulated MMP-2, MMP-9 and VEGF mRNA levels in a dose-dependent manner.

\section{Discussion}

Activation of PPAR $\gamma$ by TZDs and other ligands has been shown to inhibit proliferation and invasion, as well as induce apoptosis and cell cycle arrest in prostate and other cancer cells through PPAR $\gamma$-dependent and independent pathways (5-9). These findings suggest that activation of PPAR $\gamma$ may demonstrate anticarcinogenic potential. In the present study, it was observed that RSG was capable of downregulating CXCL12-induced migration, invasion and PI3K/Akt activation in the PC-3 PCa cell line, through inhibition of the CXCL12/CXCR4 axis. This suggests that PPAR $\gamma$ may possess antimetastatic potential.

In addition to PPAR $\gamma$-dependent effects, TZDs have been reported to induce cellular effects independent of PPAR $\gamma$ activation (15). Therefore, although the potency correlation in the present study was indicative of PPAR $\gamma$ involvement, a PPAR $\gamma$-selective antagonist was used to confirm PPAR $\gamma$ involvement. The PPAR $\gamma$ antagonist, GW9662, reduced the effect of RSG on CXCR4 expression. Therefore, these findings suggest that TZD-induced CXCR4 downregulation occurs in a PPAR $\gamma$-dependent manner. This result was in accordance with that of Richard and Blay, who demonstrated that PPAR $\gamma$ agonists inhibited CXCR4 expression in a PPAR $\gamma$-dependent manner, which was also time- and dose-dependent (16). Furthermore, pre-clinical studies have shown that the parent compound TZD is capable of reducing metastasis of HT-29 cells implanted in the rectums of mice (17). However, in a Phase II study of patients with advanced metastatic colorectal cancer that had not responded to chemotherapy, troglitazone failed to produce an objective tumor response (18). Whether treatment with a more potent and selective PPAR $\gamma$ agonist, such as RSG, is also associated with a lack of tumor response is yet to be elucidated. The present data suggest that such an agent may be capable of reducing CXCR4 expression to a greater extent, and may therefore be anticipated to demonstrate greater potential for reducing metastasis.

Metastasis is the spread of a disease from one organ or tissue to another non-adjacent organ or tissue, and is regulated by numerous signaling pathways in cancer cells and the microenvironment. The CXCR4/CXCL12 axis has a role in cancer cell metastasis and proliferation, the importance of which may vary between different types of cancer cells, due to differences in expression. For example, overexpression of CXCR4 in PCa cells has been shown to accelerate prostate tumor metastasis and vascularization, as well as tumor growth in vivo $(19,20)$. Furthermore, CXCL12 stimulates chemotaxis of metastatic PCa cells expressing high levels of CXCR4, and accelerates their migration (20). Conversely, blockade of CXCR4/CXCL12 interaction in prostate cancer cells via CXCR4 knockdown has been found to significantly inhibit bone metastasis in vivo (21).

Another important signaling pathway in PCa cells is the PI3K/Akt pathway $(22,23)$. Akt is a serine-threonine kinase whose phosphorylation is associated with mitogenic signals. In addition to its role in survival, Akt has been found to participate in numerous intracellular signaling pathways, including the integration of proliferation and differentiation signals, such as migration and angiogenesis. Previous studies have demonstrated that the PI3K/Akt pathway may also have a role in CXCL12/CXCR4-mediated PCa cell migration and angiogenesis $(24,25)$. In this study, it was observed that concomitant addition of RSG downregulated CXCL12-induced phosphorylation of Akt in a dose-dependent manner in PC-3 cells, indicating that the inhibition of migration and invasion by RSG may partly occur through suppression of PI3K pathways. In accordance with the present study, it has previously been reported that PPAR $\gamma$ agonists may be capable of decreasing Akt phosphorylation $(26,27)$. RSG was also observed to demonstrate a similar affect on insulin growth factor 1-induced phosphorylation of Akt and extracellular signal-regulated kinases in adrenocortical cancer cells (28).

In conclusion, the present data indicate that RSG is capable of inhibiting CXCL12-induced invasion and migration of PC-3 cells in vitro, through inhibition of CXCR4 expression and Akt phosphorylation. This study may provide preliminary evidence for further research into the mechanisms underlying the inhibition of metastasis by TZDs. In the present study, RSG was used only as a proof-of-principle to determine the 
efficacy of the PPAR $\gamma$ agonist class of drugs to inhibit CXCR4 expression. Based on the unclear association between RSG and risk of myocardial infarction, as well as the apparently higher risk of long-bone fracture in females that is associated with RSG, detailed safety, pharmacokinetic and pharmacodynamic studies of this class of drugs are necessary prior to clinical use in patients.

\section{Acknowledgements}

This study was supported by a grant from the Key Laboratory of Cancer Invasion and Metastasis of the Ministry of Education, China and the Ministry of Education of People's Republic of China (no. 200804871-051).

\section{References}

1. Jemal A, Siegel R, Ward E, Hao Y, Xu J and Thun MJ: Cancer statistics, 2009. CA Cancer J Clin 59: 225-249, 2009.

2. Jemal A, Bray F, Center MM, Ferlay J, Ward E and Forman D: Global cancer statistics. CA Cancer J Clin 61: 69-90, 2011.

3. Kota BP, Huang TH and Roufogalis BD: An overview on biological mechanisms of PPARs. Pharmacol Res 51: 85-94, 2005.

4. Zhou J, Zhang W, Liang B, et al: PPARgamma activation induces autophagy in breast cancer cells. Int J Biochem Cell Biol 41: 2334-2342, 2009.

5. Kim S, Lee JJ and Heo DS: PPAR $\gamma$ ligands induce growth inhibition and apoptosis through p63 and p73 in human ovarian cancer cells. Biochem Biophys Res Commun 406: 389-395, 2011.

6. Lyles BE, Akinyeke TO, Moss PE and Stewart LV: Thiazolidinediones regulate expression of cell cycle proteins in human prostate cancer cells via PPARgamma-dependent and PPARgamma-independent pathways. Cell Cycle 8: 268-277, 2009.

7. Shen D, Deng $C$ and Zhang M: Peroxisome proliferator-activated receptor gamma agonists inhibit the proliferation and invasion of human colon cancer cells. Postgrad Med J 83: 414-419, 2007.

8. Jan HJ, Lee CC, Lin YM, Lai JH, Wei HW and Lee HM: Rosiglitazone reduces cell invasiveness by inducing MKP-1 in human U87MG glioma cells. Cancer Lett 277: 141-148, 2009.

9. Fujita M, Yagami T, Fujio M, et al: Cytotoxicity of troglitazone through PPAR $\gamma$-independent pathway and p38 MAPK pathway in renal cell carcinoma. Cancer Lett 312: 219-227, 2011.

10. Liu H, Chen A, Guo F and Yuan L: A short-hairpin RNA targeting osteopontin downregulates MMP-2 and MMP-9 expressions in prostate cancer PC-3 cells. Cancer Lett 295: 27-37, 2010.

11. Zhang K, Chen D, Jiao X, et al: Slug enhances invasion ability of pancreatic cancer cells through upregulation of matrix metalloproteinase-9 and actin cytoskeleton remodeling. Lab Invest 91: 426-438, 2011.

12. Shanmugam MK, Manu KA, Ong TH, et al: Inhibition of CXCR4/CXCL12 signaling axis by ursolic acid leads to suppression of metastasis in transgenic adenocarcinoma of mouse prostate model. Int J Cancer 129: 1552-1563, 2011.
13. Yamashita D, Shimizu M and Osumi T: Mechanism for the action of PPARs. Nihon Rinsho 63: 536-537, 2005 (In Japanese).

14. Shukla S, Maclennan GT, Hartman DJ, Fu P, Resnick MI and Gupta S: Activation of PI3K-Akt signaling pathway promotes prostate cancer cell invasion. Int J Cancer 121: 1424-1432, 2007.

15. Han S and Roman J: Rosiglitazone suppresses human lung carcinoma cell growth through PPARgamma-dependent and PPARgamma-independent signal pathways. Mol Cancer Ther 5: 430-437, 2006

16. Richard CL and Blay J: Thiazolidinedione drugs down-regulate CXCR4 expression on human colorectal cancer cells in a peroxisome proliferator activated receptor gamma-dependent manner. Int J Oncol 30: 1215-1222, 2007.

17. Yoshizumi T, Ohta T, Ninomiya I, et al: Thiazolidinedione, a peroxisome proliferator-activated receptor-gamma ligand, inhibits growth and metastasis of HT-29 human colon cancer cells through differentiation-promoting effects. Int J Oncol 25: 631-639, 2004.

18. Kulke MH, Demetri GD, Sharpless NE, et al: A phase II study of troglitazone, an activator of the PPARgamma receptor, in patients with chemotherapy-resistant metastatic colorectal cancer. Cancer J 8: 395-399, 2002.

19. Darash-Yahana M, Pikarsky E, Abramovitch R, et al: Role of high expression levels of CXCR4 in tumor growth, vascularization, and metastasis. FASEB J 18: 1240-1242, 2004.

20. Arya M, Patel HR, McGurk C, et al: The importance of the CXCL12-CXCR4 chemokine ligand-receptor interaction in prostate cancer metastasis. J Exp Ther Oncol 4: 291-303, 2004.

21. Xing Y, Liu M, Du Y, et al: Tumor cell-specific blockade of CXCR4/SDF-1 interactions in prostate cancer cells by hTERT promoter induced CXCR4 knockdown: A possible metastasis preventing and minimizing approach. Cancer Biol Ther 7: 1839-1848, 2008.

22. Kreisberg JI, Malik SN, Prihoda TJ, et al: Phosphorylation of Akt (Ser473) is an excellent predictor of poor clinical outcome in prostate cancer. Cancer Res 64: 5232-5236, 2004.

23. Ayala G, Thompson T, Yang G, et al: High levels of phosphorylated form of Akt-1 in prostate cancer and non-neoplastic prostate tissues are strong predictors of biochemical recurrence. Clin Cancer Res 10: 6572-6578, 2004.

24. Wang J, Wang J, Sun Y, Song W, Nor JE, Wang CY and Taichman RS: Diverse signaling pathways through the SDF-1/CXCR4 chemokine axis in prostate cancer cell lines leads to altered patterns of cytokine secretion and angiogenesis. Cell Signal 17: 1578-1592, 2005.

25. Chinni SR, Sivalogan S, Dong Z, et al: CXCL12/CXCR4 signaling activates Akt-1 and MMP-9 expression in prostate cancer cells: the role of bone microenvironment-associated CXCL12. Prostate 66: 32-48, 2006.

26. Goetze S, Eilers F, Bungenstock A, et al: PPAR activators inhibit endothelial cell migration by targeting Akt. Biochem Biophys Res Commun 293: 1431-1437, 2002.

27. Chen WC, Lin MS and Bai X: Induction of apoptosis in colorectal cancer cells by peroxisome proliferators-activated receptor gamma activation up-regulating PTEN and inhibiting PI3K activity. Chin Med J (Engl) 118: 1477-1481, 2005.

28. Cantini G, Lombardi A, Piscitelli E, et al: Rosiglitazone inhibits adrenocortical cancer cell proliferation by interfering with the IGF-IR intracellular signaling. PPAR Res 2008: 904041, 2008. 